\title{
Negative effect of a low-carbohydrate, high-protein, high-fat diet on small peripheral artery reactivity in patients with increased cardiovascular risk
}

\author{
Jordi Merino $^{1 *}$, Richard Kones ${ }^{2}$, Raimon Ferré ${ }^{1}$, Núria Plana ${ }^{1}$, Josefa Girona ${ }^{1}$, Gemma Aragonés ${ }^{1}$, \\ Daiana Ibarretxe ${ }^{1}$, Mercedes Heras ${ }^{1}$ and Luis Masana ${ }^{1}$ \\ ${ }^{1}$ Vascular Medicine and Metabolism Unit, Lipids and Atherosclerosis Research Unit, Sant Joan University Hospital, Institut \\ d'Investigació Sanitària Pere Virgili (IISPV), Faculty of Medicine, Universitat Rovira i Virgili, Spanish Biomedical Research \\ Network in Diabetes and Associated Metabolic Disorders (CIBERDEM), Barcelona, Spain \\ ${ }^{2}$ Cardiometabolic Research Institute, 7505 Fannin Street, Suite 210, Houston, TX 77054, USA
}

(Submitted 3 February 2012 - Final revision received 27 April 2012 - Accepted 10 June 2012 - First published online 31 July 2012)

\section{Abstract}

Low-carbohydrate diets have become increasingly popular for weight loss. Although they may improve some metabolic markers, particularly in type 2 diabetes mellitus (T2D) or the metabolic syndrome (MS), their net effect on arterial wall function remains unclear. The objective was to evaluate the relation between dietary macronutrient composition and the small artery reactive hyperaemia index (saRHI), a marker of small artery endothelial function, in a cohort of patients at increased cardiovascular (CV) risk. The present cross-sectional study included 247 patients. Diet was evaluated by a 3-d food-intake register and reduced to a novel low-carbohydrate diet score (LCDS). Physical examination, demographic, biochemical and anthropometry parameters were recorded, and the saRHI was measured in each patient. Individuals in the lowest LCDS quartile (Q1, $45 \%$ carbohydrate; $20 \%$ protein; $32 \%$ fat) had higher saRHI values than those in the top quartile (Q4, 29\% carbohydrate, $24 \%$ protein, $40 \%$ fat; $1.66(\operatorname{sD~} 0.41) v .1 .52(\operatorname{SD~} 0.22), P=0.037)$. These results were particularly strong in patients with the MS $(\mathrm{Q} 1=1.82(\mathrm{sD} 0.32) v \cdot \mathrm{Q} 4=1.61(\mathrm{sD} 027) ; P=0.021)$ and T2D $(\mathrm{Q} 1=1.78(\mathrm{sD} 0.31) v \cdot \mathrm{Q} 4=1.62(\mathrm{sD} 0 \cdot 35) ; P=0 \cdot 011)$. Multivariate analysis demonstrated that individuals in the highest LCDS quartile had a significantly negative coefficient of saRHI, which was independent of confounders (OR $-0 \cdot 85 ; 95 \% \mathrm{CI} 0 \cdot 19,0 \cdot 92 ; P=0 \cdot 031)$. These findings suggest that a dietary pattern characterised by a low amount of carbohydrate, but high amounts of protein and fat, is associated with a poorer small artery vascular reactivity in patients with increased CV risk.

\section{Key words: Low-carbohydrate diets: Endothelial function: Cardiovascular risk: metabolic syndrome: Type 2 diabetes mellitus}

Obesity and the closely related risk cluster of the metabolic syndrome (MS) increase cardiovascular (CV) risk, in turn raising the incidence and prevalence of CHD. Several major scientific bodies currently recommend a low-fat, high-carbohydrate, energy-deficient diet to manage weight and associated comorbidities $^{(1-3)}$. On the other hand, diets rich in fat and protein, but low in carbohydrates, have become popular, with advocates de-emphasising the role of energy intake in weight gain. Several best-selling books endorse this strategy of carbohydrate reduction, and maintain that weight will be lost despite isoenergetic intake, accompanied by a reduction in $\mathrm{CV}$ risk. The low-carbohydrate diet score (LCDS) provides an effective tool to classify individuals according to their relative levels of fat, protein and carbohydrate consumption, embodied in a simple number ${ }^{(4)}$. The LCDS is based on the percentage of energy consumed as carbohydrate, and reflects the concordance with low carbohydrate intake. The higher the individual score, the lower the carbohydrate, but the higher the protein and fat content, in that patient's diet. The LCDS has successfully been employed to show that a low-carbohydrate diet is positively associated with the risk of type 2 diabetes (T2D) in a large cohort of healthy men after 20 years of follow-up ${ }^{(5)}$.

Measurement of the peripheral arterial tonometry (PAT) hyperaemic response, embodied in the small artery reactive hyperaemia index (saRHI), is a relatively new technique considered a toll reflecting endothelial and other variables on small artery vascular function and dilation ${ }^{(6-9)}$. The genesis, regulation and significance of changes in cutaneous digital hyperaemia differ from those affecting flow-mediated dilatation (FMD), and these determinants are not well known. We previously reported that high adherence to therapeutic lifestyle changes, including a Mediterranean dietary pattern, was associated with improved peripheral small artery vascular reactivity, as assessed by the saRHI, in conjunction with

Abbreviations: ATPIII, Adult Treatment Panel III; CV, cardiovascular; EF, endothelial function; FMD, flow-mediated dilatation; HDL-C, HDL-cholesterol; LCDS, low-carbohydrate diet score; LDL-C, LDL-cholesterol; MS, metabolic syndrome; PAT, peripheral arterial tonometry; RHI, reactive hyperaemia index; saRHI, small artery reactive hyperaemia index; T2D, type 2 diabetes mellitus.

*Corresponding author: J. Merino, fax +34 977319984, email jordi.merino@urv.cat 
improvements in carotid intima-media thickness ${ }^{(10)}$. We also generated a hypothesis that chronic consumption of high percentages of dietary protein and fat, along with a low intake of carbohydrate, may lead to peripheral small artery vascular dysfunction. The aim of the present study was to evaluate the relation between dietary macronutrient composition, using the LCDS, on the saRHI in a group of patients with increased CV risk.

\section{Subjects and methods}

\section{Design and study participants}

This is a cross-sectional study in 247 men and women, aged 30-70 years, without a previous diagnosis of CVD, who attended the Vascular Medicine and Metabolism Unit of the University Hospital Sant Joan in Reus (Spain). Only patients with intermediate to high CV risk according to the Framingham risk score, who were diagnosed with diabetes or satisfied adult treatment panel III (ATPIII) criteria for the $\mathrm{MS}^{(11)}$, were enrolled. We chose patients at intermediate to high risk primarily because re-classification of patients at this level is consistent with present practice guidelines, frequently leading to a change in therapy, and is therefore likely to be clinically meaningful and cost-effective. Patients with diagnosed CVD; chronic renal, hepatic, pulmonary or neurodegenerative problems; a history of prior neoplasia; or other serious chronic diseases were excluded. The present study was conducted according to the guidelines laid down in the Declaration of Helsinki and all procedures involving human patients were approved by the Ethical and Clinical Investigation Committee of the hospital. Written informed consent was obtained from all patients.

\section{Clinical assessment}

Each of our patients was evaluated with a review of lifestyle components (diet, physical activity and smoking), a complete physical examination including anthropometry, biochemical testing and vascular assessment with the saRHI. Physical activity was quantified in metabolic equivalent task/hours $(\mathrm{MET} / \mathrm{h})$ per week according to the Minnesota questionnaire adapted for a Spanish population ${ }^{(12)}$. Tobacco status was assessed using standardised questionnaires administered by health care providers $^{(13)}$. A 3-d food-intake register was used to evaluate the diet. The food data of dietary records were converted to energy and nutrient data by experienced dietitians and analysed using the PCN (CESNID Nutritional Program, version 2.0, University of Barcelona). The percentages of energy from carbohydrate, protein and fat were coded according to defined categories, using the LCDS method previously described by Halton et al. ${ }^{(4)}$ in their Women's Health Study Cohort. Briefly, the carbohydrate categories were scored from 10 (lowest intake) to 0 (highest intake), whereas protein and fat categories were scored from 0 (lowest intake) to 10 (highest intake). Ranks were added to create a total score with a maximum value of 30 , representing the highest intake of protein and fat and the lowest intake of carbohydrate (see Halton et al. ${ }^{(4)}$, Table 1).

\section{Peripheral small artery vascular reactivity assessment}

The saRHI was measured using PAT technology (EndoPAT-2000; Itamar Medical Limited). Determinations were performed in a quiet room with a controlled temperature $\left(22-24^{\circ} \mathrm{C}\right)$ after patients had fasted for $12 \mathrm{~h}$ and refrained from smoking or strenuous exercise for $24 \mathrm{~h}$. The patients

Table 1. Differences in demographic and lifestyle data according to low-carbohydrate diet score (LCDS) quartiles†

(Mean values and standard deviations, percentages of patients, medians and interquartile ranges)

\begin{tabular}{|c|c|c|c|c|c|c|c|c|}
\hline & \multicolumn{2}{|c|}{ LCDS Q1 (n 62) } & \multicolumn{2}{|c|}{ LCDS Q2 ( $n$ 63) } & \multicolumn{2}{|c|}{ LCDS Q3 ( $n$ 52) } & \multicolumn{2}{|c|}{ LCDS Q4 ( $n 70)$} \\
\hline & Mean & SD & Mean & SD & Mean & SD & Mean & SD \\
\hline \multicolumn{9}{|l|}{ Score (units) } \\
\hline Median & \multicolumn{2}{|c|}{14} & \multirow{2}{*}{\multicolumn{2}{|c|}{$\begin{array}{c}20 \\
1\end{array}$}} & \multirow{2}{*}{\multicolumn{2}{|c|}{$\begin{array}{c}24 \\
1\end{array}$}} & \multicolumn{2}{|c|}{27} \\
\hline Interquartile range & \multicolumn{2}{|c|}{4} & & & & & \multicolumn{2}{|c|}{1} \\
\hline Carbohydrates (\%) & $45 \cdot 56$ & 5.46 & $36 \cdot 31^{* *}$ & 4.47 & $35 \cdot 21^{\star *}$ & 3.41 & $29 \cdot 41^{\star *}$ & 4.98 \\
\hline Protein (\%) & $20 \cdot 03$ & $5 \cdot 18$ & $20 \cdot 91$ & $6 \cdot 15$ & $22 \cdot 95^{\star}$ & 3.75 & $24 \cdot 24^{\star}$ & $3 \cdot 15$ \\
\hline Fat intake (\%) & $32 \cdot 32$ & 6.02 & $40 \cdot 18^{* *}$ & $6 \cdot 76$ & $40 \cdot 79^{\star \star}$ & 5.48 & $44 \cdot 92^{\star \star}$ & $5 \cdot 02$ \\
\hline Total energy $(\mathrm{kJ} / \mathrm{d})$ & 7359 & 2133 & 7418 & 2050 & 7096 & 2665 & 6577 & 1594 \\
\hline \multicolumn{9}{|l|}{ Fibre $(g / d)$} \\
\hline Median & \multirow{2}{*}{\multicolumn{2}{|c|}{$\begin{array}{c}22 \\
7\end{array}$}} & \multirow{2}{*}{\multicolumn{2}{|c|}{20}} & \multicolumn{2}{|c|}{20} & \multicolumn{2}{|c|}{18} \\
\hline Interquartile range & & & & & \multicolumn{2}{|c|}{7} & \multicolumn{2}{|c|}{8} \\
\hline Age (years) & 55 & 11 & 56 & 10 & 54 & 9 & 54 & 9 \\
\hline Sex, men (\%) & \multicolumn{2}{|c|}{74} & \multicolumn{2}{|c|}{76} & \multicolumn{2}{|c|}{77} & \multicolumn{2}{|c|}{81} \\
\hline T2D (\%) & \multicolumn{2}{|c|}{34} & \multicolumn{2}{|c|}{26} & \multicolumn{2}{|c|}{40} & \multicolumn{2}{|c|}{37} \\
\hline Dyslipidaemia (\%) & \multicolumn{2}{|c|}{88} & \multicolumn{2}{|c|}{92} & \multicolumn{2}{|c|}{88} & & \\
\hline Metabolic syndrome (\%) & & & & & & & & \\
\hline Hypertension (\%) & & & & & & & & \\
\hline Smokers (\%) & & & & & & & & \\
\hline MET (h/week) & 33 & 28 & 32 & 25 & 26 & 16 & 23 & 20 \\
\hline Hypoglycaemic drugs (\%) & & & & & & & & \\
\hline Hypolipaemic drugs (\%) & & & & & & & & \\
\hline Antihypertensive drugs (\%) & & & & & & & & \\
\hline
\end{tabular}

T2D, type 2 diabetes mellitus; MET, metabolic equivalent task.

Mean values were significantly different from Q1: ${ }^{\star} P<0.05,{ }^{\star \star} P<0.005$

$\dagger$ Derived with an ANOVA or Wilcoxon test for continuous variables or $\chi^{2}$ test for categorical variables. 
lay in a relaxed, quiet and evenly illuminated environment while the device recorded changes in pulse waves in the digital arteries. The technique involved in PAT has been described elsewhere ${ }^{(14,15)}$. There is a $5 \mathrm{~min}$ baseline measurement, after which the cuff is inflated enough to produce ischaemia above baseline systolic pressure. After $5 \mathrm{~min}$ of occlusion, the cuff is rapidly deflated and PAT tracing is recorded for an additional $5 \mathrm{~min}$. Blood flow measurements from two fingertips - one from each hand, one a test, the other a control - were compared after a stabilisation period, and a second comparison pair of measurements was taken before and after $5 \mathrm{~min}$ of brachial ischaemia in the test arm. The results were processed by specific software to calculate the post-ischaemia reflex vasodilatation observed when measurements from the test arm (before and after ischaemia) were compared to those from the control arm. The value generated was termed the saRHI. The variability of this technique in our laboratory was $17 \%$, the intra-class correlation coefficient was 0.52 and the within-subject variation was $0 \cdot 19$.

\section{Biochemical determinations}

Venous blood samples were obtained after a 12 -h overnight fast, centrifuged immediately and stored at $-80^{\circ} \mathrm{C}$ until the assays were performed. Total cholesterol, TAG, glucose, direct LDL-cholesterol (LDL-C) and HDL-cholesterol (HDL-C), apoB100 and high sensitive C-reactive protein levels were measured using enzymatic and turbidimetric assays (Spinreact) adapted to the autoanalyser Cobas Mira Plus (Roche Diagnostics). Differential expression of human inflammatory cytokines was determined using the Human Cytokine Antibody Array (RayBiotech). The array is coated with six specific cytokine antibodies. It was probed with serum samples to allow for the comparisons of relative cytokine levels. The cytokineantibody-biotin complex was visualised with the addition of a streptavidin-labelled fluorescent dye using a laser scanner.

\section{Statistical analysis}

Data are presented as means and standard deviations or as medians and interquartile ranges for continuous variables and as frequencies for categorical variables. Normality distribution of variables was assessed with the KolmogorovSmirnov test. Differences between diet, anthropometric, biochemical or vascular data were analysed using the ANOVA or Kruskall-Wallis test for continuous variables or $\chi^{2}$ test for categorical ones. Univariate association was tested by Spearman correlation analysis. A logistic regression model test was performed to assess the determinants of the saRHI in the present study group patients. The dependent variable of interest was the highest quartile of the saRHI defined as a quartile of individuals with a high saRHI. Independent variables were selected on the basis of univariate analysis and from the variables known to be associated with the dependent variable according to our previous studies. In this test, independent variables were age, sex, T2D, atherogenic dyslipidaemia defined as low HDL-C $(<1.03 \mathrm{mmol} / 1$ in men and $1.29 \mathrm{mmol} / \mathrm{l}$ in women) and high TAG $(>1.5 \mathrm{mmol} / \mathrm{l})$, abdominal obesity and high blood pressure according to ATPIII criteria, high LDL-C ( $\geq 4 \cdot 14 \mathrm{mmol} / \mathrm{l})$, smoking, highest quartile of LCDS, highest quartile of physical activity, highest quartile of SFA intake, highest quartile of alcohol intake and highest quartile of fibre intake. $P$-values were calculated as two-sided; a $P$-value of less than 0.05 was considered statistically significant. SPSS version 19.0 (SPSS, Inc.) was used for all statistical analysis.

\section{Results}

\section{Associations of low-carbohydrate diet score with biochemical and vascular data}

The median LCDS in the present study group was 22 (SD 8) points. Participants were distributed into LCDS quartiles according to their macronutrient intake percentages (Table 1). The diet of individuals in the lowest LCDS quartile was composed of $45 \%$ carbohydrate, $20 \%$ protein and $32 \%$ fat. Participants in the top quartile had an intake of $29 \%$ carbohydrate, $24 \%$ protein and $40 \%$ fat. There were no differences between study participant quartiles other than the expected nutrient profile, as indicated in Table 1.

The differences in biochemical and vascular data between LCDS quartiles are shown in Table 2. We observed that individuals in the lowest quartile had a higher saRHI than individuals in the highest quartile (1.66 (SD 0.41) v. 1.52 (SD 0.22), $P=0.037)$. No significant differences were observed in the other vascular or biochemical parameters.

\section{Association of low-carbohydrate diet score, small artery reactive hyperaeamia index and metabolic diseases}

An inverse association was observed between the LCDS and saRHI ( $r-0 \cdot 178, P=0 \cdot 005)$. A total of 160 participants fulfilled the ATPIII criteria for the MS. The MS patients in the lowest LCDS quartile ( $n$ 38) had higher saRHI values than the MS patients in the highest LCDS quartile ( $n$ 48; 1.82 (SD 0.32) $v$. 1.61 (sD.027), $P=0.021$; Fig. 1). When the MS components were evaluated, the same association existed between each MS component, saRHI and the LCDS quartiles. The exception was in individuals with low HDL-C, in whom the saRHI was not different when comparing individuals according to the LCDS quartiles (1.74 (SD 0.35, $n$ 9) v. 1.62 (SD 0.48, $n$ 8), $P=\mathrm{NS}$ ), as illustrated in Fig. 1.

Among the eighty-four T2D patients, those in the lower quartile ( $n$ 21) had better saRHI values than those in the highest quartile ( $n$ 26; 1.78 (sD 0.31) v. 1.62 (SD 0.35), $P=0.011$; Fig. 1). These results were not modified when pharmacological therapy was taken into account.

\section{Determinants of small artery reactive hyperaemia index}

To assess the effect of LCDS on the saRHI independent of potential confounders, a multivariate stepwise test was performed. We considered the highest quartile of the saRHI (2.14 (SD 0.39) as the dependent variable and age, sex, T2D, atherogenic dyslipidaemia defined as low HDL-C 
Table 2. Anthropometric, biochemical and vascular data between lowest and highest low-carbohydrate diet score (LCDS) (Mean values and standard deviations; medians and interquartile ranges)

\begin{tabular}{|c|c|c|c|c|c|c|c|c|c|}
\hline & \multicolumn{2}{|c|}{ LCDS Q1 ( $n$ 62) } & \multicolumn{2}{|c|}{ LCDS Q2 (n 63) } & \multicolumn{2}{|c|}{ LCDS Q3 (n 52) } & \multicolumn{2}{|c|}{ LCDS Q4 ( $n 70)$} & \multirow[b]{2}{*}{$P^{*}$} \\
\hline & Mean & SD & Mean & SD & Mean & SD & Mean & SD & \\
\hline Body weight $(\mathrm{kg})$ & 87.9 & 14 & 88.6 & 13.5 & $87 \cdot 1$ & $13 \cdot 6$ & $89 \cdot 4$ & $13 \cdot 1$ & 0.819 \\
\hline Waist circumference $(\mathrm{cm})$ & 105 & 9 & 105 & 9 & 104 & 9 & 105 & 8 & 0.831 \\
\hline Systolic blood pressure $(\mathrm{mmHg})$ & & & & & & & & & 0.561 \\
\hline Median & \multirow{2}{*}{\multicolumn{2}{|c|}{130}} & \multicolumn{2}{|c|}{132} & \multicolumn{2}{|c|}{130} & \multicolumn{2}{|c|}{135} & \\
\hline Interquartile range & 50 & & \multicolumn{2}{|c|}{15} & \multicolumn{2}{|c|}{36} & \multicolumn{2}{|c|}{30} & \\
\hline Total cholesterol $(\mathrm{mmol} / \mathrm{l})$ & 5.43 & 1.54 & $5 \cdot 14$ & 1.04 & 5.29 & 1.08 & $5 \cdot 26$ & 1.08 & 0.295 \\
\hline LDL-C $(\mathrm{mmol} / \mathrm{l})$ & 3.34 & 0.98 & 3.04 & 0.98 & 3.41 & 1.02 & 3.23 & 0.87 & 0.128 \\
\hline $\mathrm{HDL}-\mathrm{C}(\mathrm{mmol} / \mathrm{l})$ & 1.36 & 0.37 & 1.35 & 0.27 & 1.36 & 0.29 & 1.31 & 0.37 & 0.889 \\
\hline apoB100 (mmol/l) & $1 \cdot 18$ & 0.23 & $1 \cdot 18$ & 0.21 & 1.19 & 0.24 & 1.17 & 0.23 & 0.199 \\
\hline TAG $(\mathrm{mmol} / \mathrm{l})$ & & & & & & & & & 0.180 \\
\hline Median & \multicolumn{2}{|c|}{1.91} & \multicolumn{2}{|c|}{$2 \cdot 16$} & \multicolumn{2}{|c|}{2.09} & \multicolumn{2}{|c|}{2.49} & \\
\hline Interquartile range & \multicolumn{2}{|c|}{0.97} & \multicolumn{2}{|c|}{1.44} & \multicolumn{2}{|c|}{$1 \cdot 21$} & \multicolumn{2}{|c|}{2.05} & \\
\hline Glucose (mmol/l) & & & & & & & & & 0.444 \\
\hline Median & \multicolumn{2}{|c|}{$5 \cdot 76$} & \multicolumn{2}{|c|}{5.88} & \multicolumn{2}{|c|}{5.92} & \multicolumn{2}{|c|}{$6 \cdot 19$} & \\
\hline Interquartile range & & & & & & & & & \\
\hline $\mathrm{hs}-\mathrm{CRP}(\mathrm{mmol} / \mathrm{l})$ & & & & & & & & & 0.515 \\
\hline Median & & & & & & & & & \\
\hline Interquartile range & & & & & & & & & \\
\hline IL-6 (pg/ml) & & & & & & & & & 0.434 \\
\hline Median & & & & & & & & & \\
\hline Interquartile range & & & & & & & & & \\
\hline $\mathrm{TNF}-\alpha(\mathrm{pg} / \mathrm{ml})$ & & & & & & & & & 0.343 \\
\hline Median & & & & & & & & & \\
\hline Interquartile range & & & & & & & & & \\
\hline sICAM-1 (ng/ml) & & & & & & & & & 0.621 \\
\hline Median & & & & & & & & & \\
\hline Interquartile range & & & & & & & & & \\
\hline sVCAM-1 $(\mathrm{ng} / \mathrm{ml})$ & & & & & & & & & 0.609 \\
\hline Median & & & & & & & & & \\
\hline Interquartile range & & & & & & & & & \\
\hline $\mathrm{sE}$-selectin $(\mathrm{ng} / \mathrm{ml})$ & & & & & & & & & 0.407 \\
\hline Median & & & & & & & & & \\
\hline Interquartile range & & & & & & & & & \\
\hline saRHI (units) & & & & & & & & & 0.037 \\
\hline Median & & & & & & & & & \\
\hline Interquartile range & & & & & & & & & \\
\hline
\end{tabular}

$(<1.03 \mathrm{mmol} / 1$ in men and $1.29 \mathrm{mmol} / 1$ in women $)$ and high TAG $(>1.5 \mathrm{mmol} / \mathrm{l})$, abdominal obesity and high blood pressure according to ATPIII criteria, high LDL-C $(\geq 4 \cdot 14 \mathrm{mmol} / \mathrm{l})$, smoking, highest quartile of LCDS, highest quartile of physical activity (40 (SD 18) MET/h per week), highest quartile of SFA intake $(11.6(\mathrm{SD} 2 \cdot 8) \mathrm{g} / \mathrm{d})$, highest quartile of alcohol intake $(21(\mathrm{SD} 12) \mathrm{g} / \mathrm{d})$ and highest quartile of fibre intake $(22(\mathrm{SD} 7) \mathrm{g} / \mathrm{d})$ as independent variables. As seen in Fig. 2, individuals in the highest LCDS quartile, as compared with the lowest quartile, had negative probabilities of high saRHI, with a estimated prognostic value of $75 \%$ (OR $-0 \cdot 85 ; 95 \%$ CI $0 \cdot 19$, $0.92 ; P=0.031)$. Saturated fat intake was not an independent predictor of saRHI (OR $-0 \cdot 88 ; 95 \%$ CI: $0 \cdot 41,1 \cdot 93 ; P=0 \cdot 631)$.

\section{Discussion}

Stratifying participants according to their unique dietary macronutrient composition (LCDS), we examined the impact of varying dietary carbohydrate content on small artery vascular function assessed by a recently homologated new tool, the saRHI. We observed that the higher the LCDS, reflecting a lower dietary carbohydrate content, the lower the small artery reactivity in the digital vascular bed. This inverse relationship was significant and independent of the analyses used and a characteristic of the metabolic risk factors, especially in patients with T2D, the MS or abdominal obesity. While differences in endothelial function (EF) biomarkers did not reach statistical significance, they varied in the same direction. In essence, patients consuming carbohydrate-poor diets had the worst EF profiles.

The literature suggests that diets with low or very low carbohydrate content may be associated with impaired small artery vascular function ${ }^{(16-19)}$. Randomised clinical trials have shown that weight loss per se is the main determinant of improvement in peripheral small artery reactivity using a low-carbohydrate diet or low-fat diet ${ }^{(20-25)}$. In fact, obesity and visceral adiposity are associated with endothelial dysfunction in resistance and conduit arteries, and weight loss brought about by lowered energy intake improves EF, even in the absence of heart disease or CV risk factors ${ }^{(26,27)}$. 


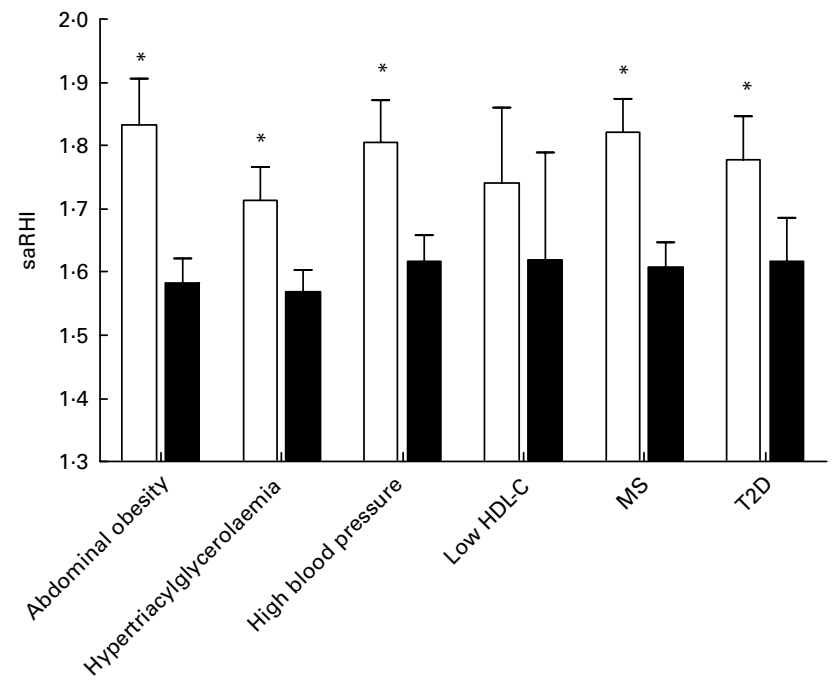

Fig. 1. Differences in the small artery reactive hyperaemia index (saRHI) according to low-carbohydrate diet score (LCDS) quartile and the cardiovascular risk factors. $\square$, LCDS quartile 1 patients; $\mathbf{n}$, LCDS quartile 4 patients. Data obtained with Kruskall-Wallis test. Values are medians with their interquartile ranges. * Median values were significantly different $(P<0.05)$. HDL-C, HDL-cholesterol; MS, metabolic syndrome; T2D, type 2 diabetes mellitus.

However, in clinical trials without changes in body weight, low-carbohydrate/high-protein diets may be significantly associated with twice the amount of arterial plaque, a reduction in the number of circulating and bone marrow endothelial progenitor cells in murine models of atherosclerosis $^{(28)}$ and lower FMD in healthy and obese subjects ${ }^{(29)}$. One dissenting randomised clinical trial included forty obese and overweight subjects with hypertriacylglycerolaemia, showing an inverse association between carbohydrates and FMD.
However, these data were collected postprandially and cannot be directly extrapolated to basal conditions ${ }^{(30)}$. The present results therefore reinforce the known negative impact of very low-carbohydrate diets on vascular function assessed by FMD under fasting conditions, using the PAT technique.

The components and variables affecting EF, assessed by FMD, as compared with PAT differ, and although there may be some correlation, the two techniques provide distinct information about vascular function in conduit arteries $v$. the peripheral small arteries of the finger ${ }^{(31)}$

The FMD is highly dependent on NO production, whereas the reactive hyperaemia index (RHI) is not, even though NO has been reported to contribute to $\mathrm{RHI}^{(8,32)}$. While the European Society of Cardiology classifies RHI with other tech-

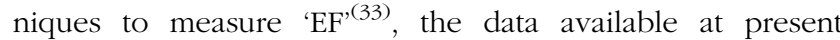
indicate that each of these methods probably has a unique blend of physiological determinants, and each provides particular information about different vascular beds ${ }^{(31,34)}$. A recent direct comparison of methods did not reveal a strong correlation ${ }^{(9)}$. However, FMD is difficult to perform compared to the RHI, and the correlation between the two and other data derived are sufficient to continue clinical investigation $^{(9,34)}$. Indeed, the saRHI correlates with coronary endothelial dysfunction ${ }^{(14)}$, accurately measures vascular effects when the net CV risk burden improves ${ }^{(35)}$, correlates with carotid intima-media thickness and can predict future adverse $\mathrm{CV}$ events ${ }^{(36)}$. Information from non-invasive PAT studies is useful to assess static or serial vascular function in a variety of patient phenotypes with raised $\mathrm{CV}$ risk $^{(37)}$.

Our group has shown that saRHI-measured endothelial dysfunction is also associated with increased soluble E-selectin plasma concentrations, suggesting that at least

Highest saRHI quartile

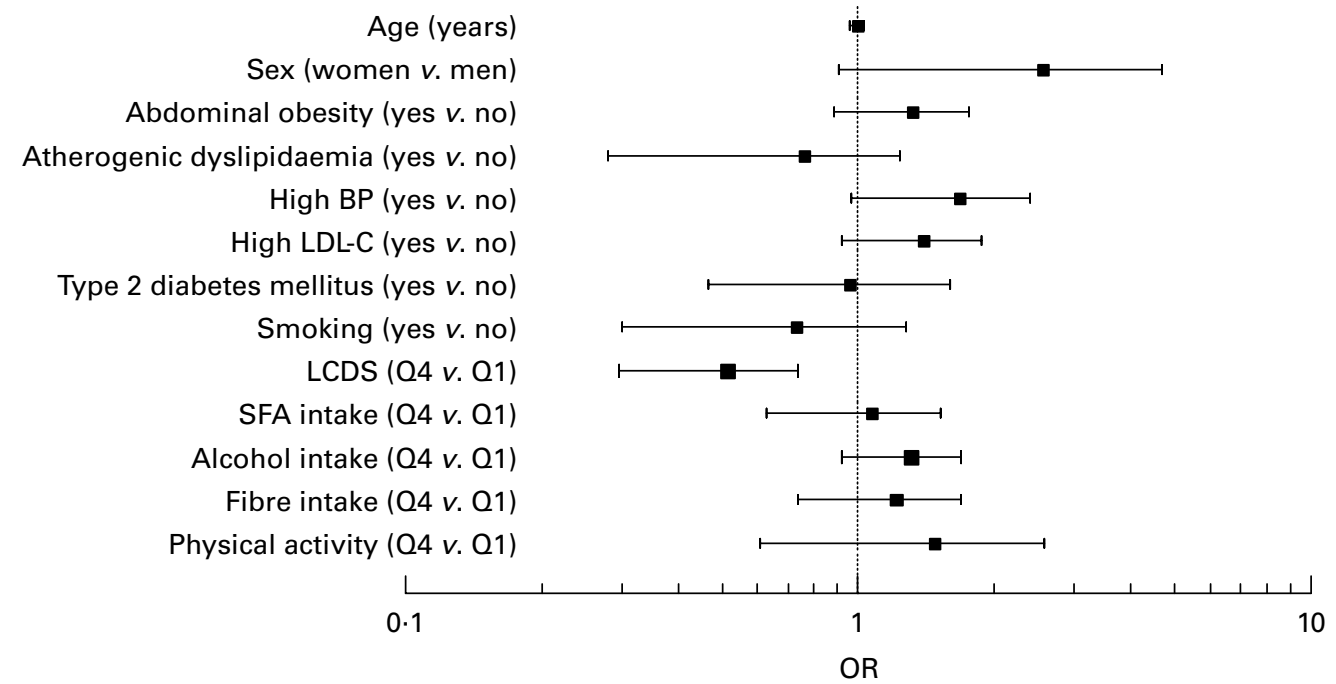

Fig. 2. Determinants of small artery reactive hyperaemia index (saRHI). Multivariate stepwise binary logistic regression test. Dependent variable: highest quartile of saRHI (2.14 (SD 0.39)). Independent variables: age, sex, atherogenic dyslipidaemia (TAG $>1.5 \mathrm{mmol} / \mathrm{l}$ and $\mathrm{HDL}-\mathrm{cholesterol}<1.03 \mathrm{mmol} / \mathrm{l}$ in men and $1.29 \mathrm{mmol} / \mathrm{l}$ in women), abdominal obesity and high blood pressure (BP) according to adult treatment panel III criteria, high LDL-cholesterol (LDL-C $\geq 4.14 \mathrm{mmol} / \mathrm{l}$ ), smoking, highest quartile of low-carbohydrate diet score (LCDS), highest quartile of physical activity (40 (SD 18) metabolic equivalent task/h per week), highest quartile of SFA intake (11.6 (SD 2.8) g/d), highest quartile of alcohol intake (21 (SD 12) g/d) and highest quartile of fibre intake (22 (SD 7) g/d). Estimated prognostic value: $75 \% . R^{2}$ Nagelkerke: 0.62 . 
some mechanisms are shared by both large and small artery function $^{(38)}$. In patients without the MS but with risk-related elevations in soluble E-selectin and soluble vascular adhesion molecule 1, there was a strong correlation between saRHI values and levels of oxidised $\mathrm{LDL}^{(39)}$. A uniting theme is that the saRHI is clearly associated with metabolic parameters, mainly waist circumference, and plasma concentrations of TAG, HDL-C and apoA $1^{(40)}$. In the present study, we observed significant differences in saRHI between LCDS quartiles composed of hypertriacylglycerolaemic and abdominal obesity subjects; a lack of a similar relationship in subjects with low HDL-C might be due to the small sample size of patients with low HDL-C criteria (Q1 $=9$ and Q4=8).

\section{Limitations}

The present study had other limitations. One was related to the score used in the study and the cut-off values. The LCDS was performed using US population data, and the percentiles of diet relate to healthy American white men and women. However, as the diet consumed by the present study population was similar to the diet of a corresponding American population with equally high $\mathrm{CV}$ risk $^{(41)}$, the comparison is reasonable. We did not grade or independently consider dietary amounts of animal or vegetable protein or dietary fat, as the primary focus was the influence of low carbohydrate intake on small artery vascular reactivity. As the study was conducted in patients with increased CV risk, results cannot be generalised to healthy individuals. The present study design did not control all possible confounding variables. Errors associated with completion of food diaries are possible. Although not described, either the pharmacological agents that our patients were taking themselves, or interaction with macronutrient changes, could have influenced EF. Uncontrolled dietary ingredients, such as $n$ - 3 fatty acids and polyphenols, could potentially confound the results. Finally, a cross-sectional study cannot establish causality. In view of the strength of our main finding, the quantitative importance of these limitations appears small.

\section{Conclusion}

In a cross-sectional study of patients with increased CV risk, a dietary pattern characterised by a high LCDS (high protein and fat, but low carbohydrate content) was associated with poorer peripheral small artery function compared with individuals consuming a diet with a lower LCDS. The association was strong in patients with two different metabolic diseases studied: the MS and T2D. This relationship needs confirmation with larger cohorts in randomised clinical trials.

\section{Acknowledgements}

The present work was supported by grants from ISCIII, Madrid, Spain (PI 05/1954). CIBERDEM is an initiative of ISCIII, Spain. The authors declared no conflict of interest. The authors' responsibilities were as follows: R. F., N. P. and L. M. designed the study; J. M., G. A., R. F. and N. P. conducted research; M. H. performed the biochemical analyses; J. M., R. F. and L. M. performed the statistical tests and wrote the final manuscript. R. K. reviewed the manuscript, and contributed significantly to the final form of the introduction and the discussion. All authors read and approved the final manuscript.

\section{References}

1. Krauss RM, Eckel RH, Howard B, et al. (2000) AHA Dietary Guidelines: revision 2000: a statement for healthcare professionals from the Nutrition Committee of the American Heart Association. Stroke 31, 2751-2766.

2. Cummings S, Parham ES \& Strain GW (2002) Position of the American Dietetic Association: weight management. $J \mathrm{Am}$ Diet Assoc 102, 1145-1155.

3. Flock MR \& Kris-Etherton PM (2011) Dietary Guidelines for Americans 2010: implications for cardiovascular disease. Curr Atheroscler Rep 13, 499-507.

4. Halton TL, Willett WC, Liu S, et al. (2006) Low-carbohydratediet score and the risk of coronary heart disease in women. $N$ Engl J Med 355, 1991-2002.

5. de Koning L, Fung TT, Liao X, et al. (2011) Low-carbohydrate diet scores and risk of type 2 diabetes in men. Am J Clin Nutr 93, 844-850.

6. Kuvin JT, Patel AR, Sliney KA, et al. (2003) Assessment of peripheral vascular endothelial function with finger arterial pulse wave amplitude. Am Heart J 146, 168-174.

7. Nohria A, Gerhard-Herman M, Creager MA, et al. (2006) Role of nitric oxide in the regulation of digital pulse volume amplitude in humans. $J$ Appl Physiol 101, 545-548.

8. Celermajer DS (2008) Reliable endothelial function testing at our fingertips? Circulation 117, 2428-2430.

9. Dhindsa M, Sommerlad SM, DeVan AE, et al. (2008) Interrelationships among noninvasive measures of postischemic macro- and microvascular reactivity. J Appl Physiol 105, 427-432.

10. Ferre R, Plana N, Merino J, et al. (2012) Effects of therapeutic lifestyle changes on peripheral artery tonometry in patients with abdominal obesity. Nutr Metab Cardiovasc Dis 22, 95-102.

11. Grundy SM, Cleeman JI, Daniels SR, et al. (2005) Diagnosis and management of the metabolic syndrome: an American Heart Association/National Heart, Lung, and Blood Institute scientific statement: Executive Summary. Crit Pathw Cardiol 4, 198-203.

12. Elosua R, Garcia M, Aguilar A, et al. (2000) Validation of the Minnesota leisure time physical activity questionnaire in Spanish women. Investigators of the MARATDON Group. Med Sci Sports Exerc 32, 1431-1437.

13. DiClemente CC, Prochaska JO, Fairhurst SK, et al. (1991) The process of smoking cessation: an analysis of precontemplation, contemplation, and preparation stages of change. J Consult Clin Psychol 59, 295-304.

14. Bonetti PO, Pumper GM, Higano ST, et al. (2004) Noninvasive identification of patients with early coronary atherosclerosis by assessment of digital reactive hyperemia. J Am Coll Cardiol 44, 2137-2141.

15. Hamburg NM, Keyes MJ, Larson MG, et al. (2008) Cross-sectional relations of digital vascular function to cardiovascular risk factors in the Framingham Heart Study. Circulation 117, 2467-2474.

16. Dod HS, Bhardwaj R, Sajja V, et al. (2010) Effect of intensive lifestyle changes on endothelial function and on inflammatory markers of atherosclerosis. Am J Cardiol 105, 362-367. 
17. Miller M, Beach V, Sorkin JD, et al. (2009) Comparative effects of three popular diets on lipids, endothelial function, and C-reactive protein during weight maintenance. J Am Diet Assoc 109, 713-717.

18. Wycherley TP, Brinkworth GD, Keogh JB, et al. (2010) Long-term effects of weight loss with a very low carbohydrate and low fat diet on vascular function in overweight and obese patients. J Intern Med 267, 452-461.

19. Phillips SA, Jurva JW, Syed AQ, et al. (2008) Benefit of low-fat over low-carbohydrate diet on endothelial health in obesity. Hypertension 51, 376-382.

20. Foster GD, Wyatt HR, Hill JO, et al. (2003) A randomized trial of a low-carbohydrate diet for obesity. $N$ Engl J Med 348, 2082-2090.

21. Gardner CD, Kiazand A, Alhassan S, et al. (2007) Comparison of the Atkins, Zone, Ornish, and LEARN diets for change in weight and related risk factors among overweight premenopausal women: the A to Z Weight Loss Study: a randomized trial. JAMA 297, 969-977.

22. Johnstone AM, Lobley GE, Horgan GW, et al. (2011) Effects of a high-protein, low-carbohydrate $v$. high-protein, moderate-carbohydrate weight-loss diet on antioxidant status, endothelial markers and plasma indices of the cardiometabolic profile. BrJ Nutr 106, 282-291.

23. Clifton PM, Keogh JB, Foster PR, et al. (2005) Effect of weight loss on inflammatory and endothelial markers and FMD using two low-fat diets. Int $J$ Obes (Lond) 29, $1445-1451$.

24. Keogh JB, Brinkworth GD, Noakes M, et al. (2008) Effects of weight loss from a very-low-carbohydrate diet on endothelial function and markers of cardiovascular disease risk in subjects with abdominal obesity. Am J Clin Nutr 87, 567-576.

25. Ferrara LA, Innelli P, Palmieri $\mathrm{V}$, et al. (2006) Effects of different dietary protein intakes on body composition and vascular reactivity. Eur J Clin Nutr 60, 643-649.

26. Pierce GL, Beske SD, Lawson BR, et al. (2008) Weight loss alone improves conduit and resistance artery endothelial function in young and older overweight/obese adults. Hypertension 52, 72-79.

27. Brook RD (2006) Obesity, weight loss, and vascular function. Endocrine 29, 21-25.

28. Foo SY, Heller ER, Wykrzykowska J, et al. (2009) Vascular effects of a low-carbohydrate high-protein diet. Proc Natl Acad Sci U S A 106, 15418-15423.

29. Phillips SA (2007) Effects of low-carbohydrate diet on vascular health: more than just weight loss. Am J Physiol Heart Circ Physiol 292, H2037-H2039.
30. Volek JS, Ballard KD, Silvestre R, et al. (2009) Effects of dietary carbohydrate restriction versus low-fat diet on flow-mediated dilation. Metabolism 58, 1769-1777.

31. Hamburg NM, Palmisano J, Larson MG, et al. (2011) Relation of brachial and digital measures of vascular function in the community: The Framingham Heart Study. Hypertension 57, 390-396.

32. Bacon SL, Lavoie KL, Arsenault A, et al. (2011) The research on endothelial function in women and men at risk for cardiovascular disease (REWARD) study: methodology. BMC Cardiovasc Disord 11, 50.

33. Lekakis J, Abraham P, Balbarini A, et al. (2011) Methods for evaluating endothelial function: a position statement from the European Society of Cardiology Working Group on Peripheral Circulation. Eur J Cardiovasc Prev Rehabil $\mathbf{1 8}$ 775-789.

34. Schnabel RB, Schulz A, Wild PS, et al. (2011) Noninvasive vascular function measurement in the community: crosssectional relations and comparison of methods. Circ Cardiovasc Imaging 4, 371-380.

35. Chouraqui P, Schnall RP, Dvir I, et al. (2002) Assessment of peripheral artery tonometry in the detection of treadmill exercise-induced myocardial ischemia. J Am Coll Cardiol 40, 2195-2200.

36. Rubinshtein R, Kuvin JT, Soffler M, et al. (2010) Assessment of endothelial function by non-invasive peripheral arterial tonometry predicts late cardiovascular adverse events. Eur Heart J 31, 1142-1148.

37. Plana N, Ferre R, Merino J, et al. (2011) Heterozygous familial hypercholesterolaemic patients have increased arterial stiffness, as determined using the augmentation index. $J$ Atheroscler Thromb 18, 1110-1116.

38. Versari D, Daghini E, Virdis A, et al. (2009) Endothelial dysfunction as a target for prevention of cardiovascular disease. Diabetes Care 32, Suppl. 2, S314-S321.

39. Aragones G, Ferre R, Girona J, et al. (2012) Small artery dilation and endothelial markers in cardiovascular risk patients. Eur J Clin Invest 42, 34-41.

40. Ferre R, Aragones G, Plana N, et al. (2011) High-density lipoprotein cholesterol and apolipoprotein $\mathrm{A}_{1}$ levels strongly influence the reactivity of small peripheral arteries. Atherosclerosis 216, 115-119.

41. Yeboah J, Crouse JR, Hsu FC, et al. (2007) Brachial flowmediated dilation predicts incident cardiovascular events in older adults: the Cardiovascular Health Study. Circulation 115, 2390-2397. 